\title{
Preoperative external tissue expansion for complex cranial reconstructions
}

\author{
Kevin A. Reinard, MD, ${ }^{1}$ Hesham M. Zakaria, MD, ${ }^{1}$ Ahmad Qatanani, BS, ${ }^{2}$ Ian Y. Lee, MD, ${ }^{1}$ \\ Jack P. Rock, MD, ${ }^{1}$ and Herman P. Houin, MD ${ }^{3}$ \\ Departments of ${ }^{1}$ Neurosurgery and ${ }^{3}$ Plastic Surgery, Henry Ford Hospital, Detroit, Michigan; and ${ }^{2}$ Department of Biomedical \\ Engineering, New Jersey Technical Institute, Newark, New Jersey
}

\begin{abstract}
OBJECTIVE Reconstruction of large solitary cranial defects after multiple craniotomies is challenging because scalp contraction generally requires more than simple subcutaneous undermining to ensure effective and cosmetically appealing closure. In plastic and reconstructive surgery, soft tissue expansion is considered the gold standard for reconstructing scalp defects; however, these techniques are not well known nor are they routinely practiced among neurosurgeons. The authors here describe a simple external tissue expansion technique that is associated with low morbidity and results in high cosmetic satisfaction among patients.

METHODS The authors reviewed the medical records of patients with large cranial defects $(>5 \mathrm{~cm})$ following multiple complicated craniotomies who had undergone reconstructive cranioplasty with preoperative tissue expansion using the DermaClose RC device. In addition to gathering data on patient age, sex, primary pathology, number of craniotomies and/or craniectomies, history of radiation therapy, and duration of external scalp tissue expansion, the authors screened patient charts for cerebrospinal fluid (CSF) leak, meningitis, intracranial abscess formation, dermatitis, and patient satisfaction rates.
\end{abstract}

RESULTS The 6 identified patients ( 5 female, 1 male) had an age range from 36 to 70 years. All patients had complicating factors such as recalcitrant scalp infections after multiple craniotomies or cranial radiation, which led to secondary scalp tissue scarring and retraction. All patients were deemed to be potential candidates for rotational flaps with or without skin grafts. All patients underwent the same preoperative tissue expansion followed by standard cranial bone reconstruction. None of the patients developed CSF leak, meningitis, intracranial abscess, dermatitis, or permanent cosmetic defects. None of the patients required a reoperation. Mean follow-up was 117 days.

CONCLUSIONS Preoperative scalp tissue expansion with the DermaClose RC device allows for simple and reliable completion of complicated cranial reconstruction with low morbidity rates and high cosmetic satisfaction among patients. http://thejns.org/doi/abs/10.3171/2015.7.JNS15132

KEY WORDS craniotomy; craniectomy; reconstructive surgery; surgical technique

$\mathrm{M}$ ULTIPLE craniotomies and craniectomies at a solitary site are not uncommon in neurosurgical practice and are associated with high morbidity. A number of clinical indications support the practice of craniectomy ${ }^{6,31}$ across a wide age group. ${ }^{8}$ After craniectomies, the bone flap must be replaced with cranioplasty to prevent neurological susceptibility to a skull defect. ${ }^{14}$ To help reduce scalp volume contraction, early cranioplasty can be considered; however, the timing of cranioplasty remains controversial. ${ }^{26}$ Several recent reports have advocated early cranioplasty to improve neurological outcome following this potentially morbid procedure. ., $, 22,29,32$ Even though various cranioplasty techniques have been described throughout history, $1,4,5,12,16,21$ tissue expansion for patients who require complex cranial reconstruction was introduced by Neumann ${ }^{19}$ in 1957 and has since become the gold standard of practice for plastic surgeons. ${ }^{15}$ However, only a few reports have provided evidence-based examples of the use of scalp tissue expansion prior to cranioplasty in complicated cases, and there is no consensus guideline for the repair of these complex cranial reconstructions..$^{13,16,18}$ To provide more options for neu-

ABBREVIATIONS DSSA = defective scalp surface area; DSV = defective scalp volume; EBRT = external beam radiation therapy; SSSA = stretched scalp surface area; SSV = stretched scalp volume. 
rosurgeons and plastic surgeons performing complicated cranioplasties, we describe a novel yet simple technique for scalp tissue expansion that offers excellent and reproducible aesthetic outcomes with low associated morbidity.

\section{Methods}

With approval from the Henry Ford Hospital Institutional Review Board, we searched our patient database and identified patients who had undergone definitive cranioplasty with preoperative scalp tissue expansion using the DermaClose RC device (Wound Care Technologies Inc.; http://dermaclose.com/). Electronic medical records were searched to gather patient demographic data, including age, sex, primary pathology, number of craniotomies and/or craniectomies, history of radiation therapy, duration of external scalp tissue expansion, and patient discomfort level after application of the DermaClose RC device. Patient charts were analyzed for structural success rates as well as complications related to the definitive cranioplasty.

Only patients with a history of multiple $(\geq 3)$ craniotomies and/or craniectomies with associated complicating factors, such as prior cranial radiation or recalcitrant scalp infections, were considered candidates for DermaClose $\mathrm{RC}$ device application. In all cases, the neurosurgeon and the plastic surgeon concurred that significant tissue scarring and scalp atrophy necessitated tissue expansion to ensure tension-free skin closure. All patients underwent delayed placement of the DermaClose RC tissue expander device by a plastic surgeon, either in the outpatient setting or in the operating room under local anesthesia.

\section{Technique}

The DermaClose RC has 10 anchors that pierce the dermis with traction clips, which are then secured with staples. These anchors, which the traction line tabs set approximately $1 \mathrm{~cm}$ outside the bone defect margin, are spaced circumferentially around the cranial defect. A tension controller is then sutured to the scalp in a position that will not inhibit the patient's favorite sleeping position. Next, the tension line is placed circumferentially through the tension line tabs of each anchor. A tension line placed circumferentially rather than in a crisscrossing pattern usually conforms more naturally to the curved scalp. The underlying skin is protected by placing customized, longitudinally slit plastic tubes over the tension lines (Fig. 1).

On average, the DermaClose RC device was applied to patients 238 days ( $\mathrm{SD} \pm 60$ days) after their last craniectomy, when maximal scalp tissue retraction had occurred. All patients were clinically deemed to be appropriate candidates for rotational flaps with or without skin grafts. Over the course of 7-10 days, the plastic surgeon sequentially adjusted the device to apply constant tension to the scalp tissue, which facilitates tissue expansion to provide more skin surface area for definitive closure. ${ }^{9}$ The DermaClose RC device automatically maintains tension below capillary filling pressure, which is $30 \mathrm{~mm} \mathrm{Hg}$.

\section{Surface Area and Scalp Volume Calculations}

Thin-cut head CT scans were used in conjunction with computerized $3 \mathrm{D}$ rendering software to generate a $3 \mathrm{D}$

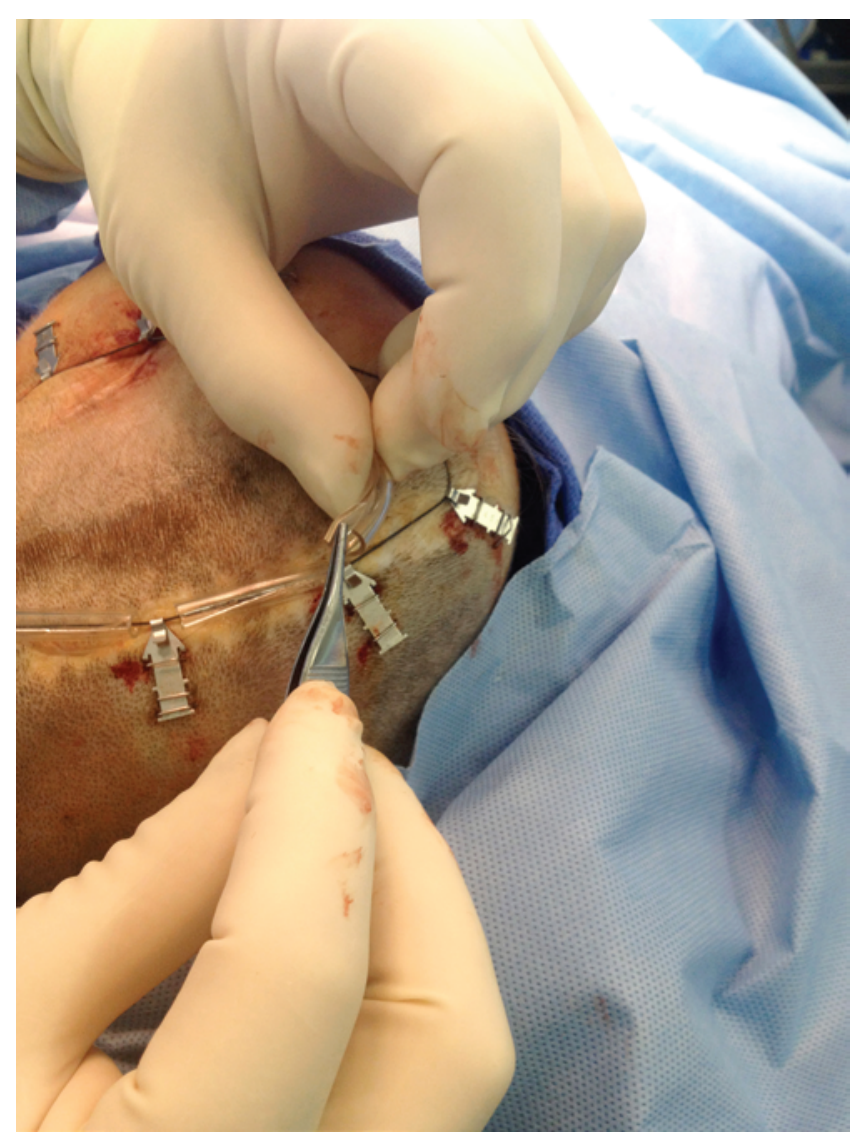

FIG. 1. Application of the DermaClose RC device under local anesthesia. The anchors and traction clips are set $1 \mathrm{~cm}$ outside the defect margin. Notice the tension line that is placed circumferentially through each anchor within plastic tubes that protect the scalp from laceration. Figure is available in color online only.

model of each patient's cranial soft tissues and defect (Fig. 2A). The thickness of soft tissue on each patient's contralateral skull, which is normally $4-6 \mathrm{~mm}$, was then estimated on the CT scan (Fig. 2B). Next, the initial implant, designed to mirror contralateral symmetry, was expanded in the external direction by the absolute value of the thickness of the soft tissue overlying the contralateral virgin skull. We then arbitrarily adjusted the tissue thickness subjacent to the implant (Fig. 2C) such that this hybrid configuration snugly fit into the patient's original 3D defect model and produced a composite volume model (Fig. 2D). By subtracting the composite volume model from the original 3D soft tissue model, the computer software was able to estimate the total expanded tissue surface area and volumes (Fig. 2E).

To more accurately estimate the efficacy of the soft tissue expansion achieved with the application of the DermaClose RC device, we also calculated the scalp surface area prior to expansion (defective scalp surface area [DSSA]) by simply delineating the margins of the scalp around the cranial defect and allowing the computer software to automatically calculate the area within the curve. Because none of the patients underwent repeat CT imaging after application of the DermaClose RC device, information regarding the stretched scalp surface area after device appli- 

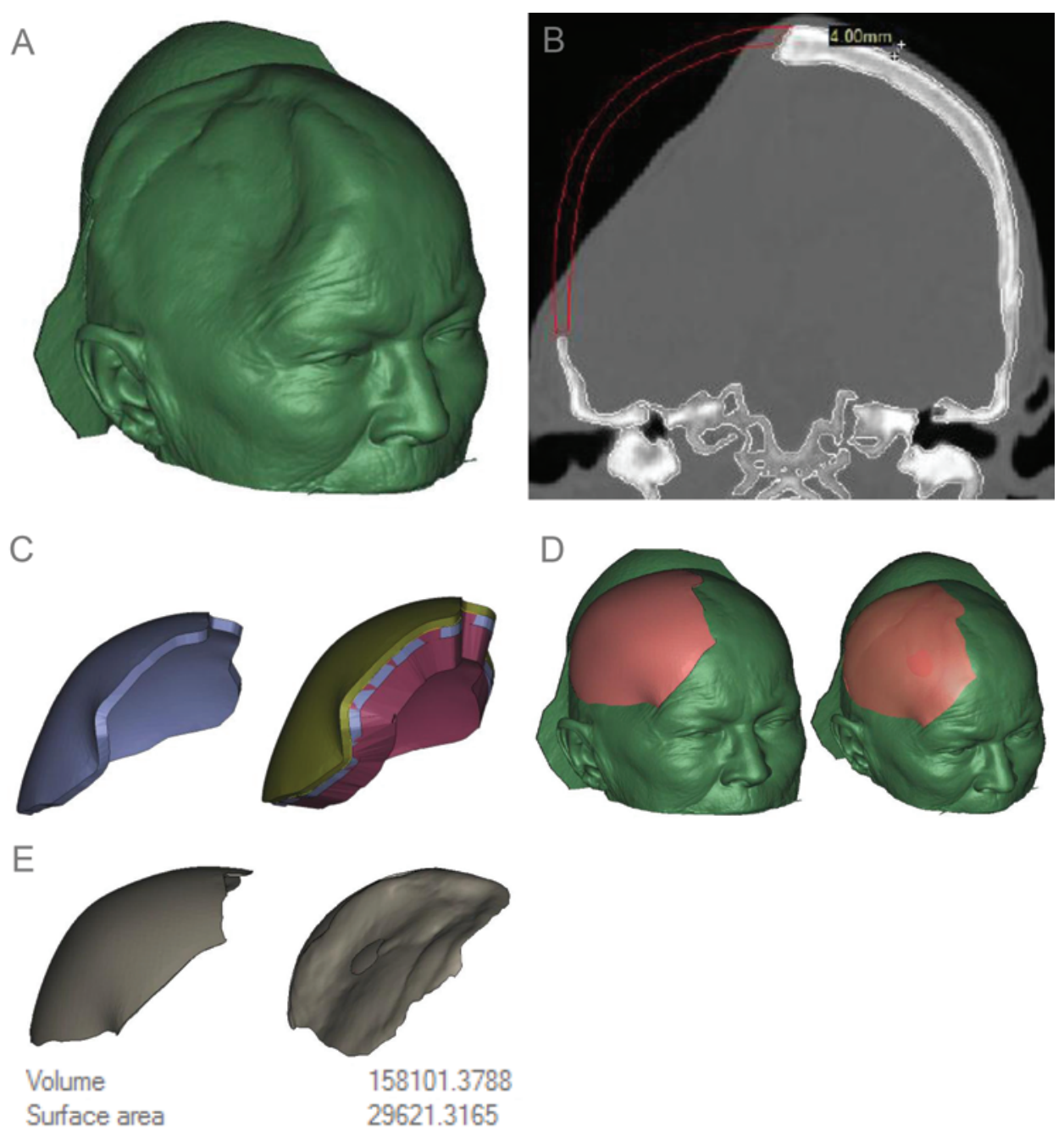

FIG. 2. Estimation of the expanded soft tissue volumes. A: Three-dimensional model of a patient's cranial soft tissues demonstrating the defect contour. B: Estimate of the scalp thickness on the contralateral side as well as the approximate contour of the cranioplasty plate. C: The cranioplasty plate (left) and the composite volume model (right; green cap indicates the contralateral virgin skull, and purple cap indicates tissue subjacent to the implant). D: The patient's ideal soft tissue contour. E: Estimated surface area of the expanded tissue as well as its volume. Figure is available in color online only.

cation was extrapolated from the aforementioned volume model (Fig. 2D) by placing a curvilinear line just above where the cranial defect margins would be (stretched scalp surface area [SSSA]). Scalp volumes prior to tissue expansion (defective scalp volume [DSV]) and after tissue expansion (stretched scalp volume [SSV]) were calculated by extruding the surface area measurements described above and using the estimated thickness of the scalp soft tissue on the contralateral side. Using the measured DSSA and SSSA as well as the DSV and SSV, we were able to calculate percentage increases in scalp surface area and volume achieved by the DermaClose RC device

All patients were maintained on low doses of oral narcotic-based analgesics (5-7.5 mg hydrocodone/acetaminophen, Norco) while undergoing scalp expansion. Once adequate scalp expansion was confirmed by the plastic surgeon, all patients underwent delayed but definitive cranioplasty (Fig. 3) with a tension-free skin closure. A Jackson-Pratt drain was placed subgaleally in all patients to minimize fluid collection and reduce the risk of postop- erative infection. A postoperative CT scan was obtained in every patient. All patients were discharged an average of 3.3 days ( $\mathrm{SD} \pm 2$ days) after cranioplasty. No perioperative complications were encountered in any of the patients.

\section{Results}

We performed cranioplasties in 6 patients, 5 female $(83 \%)$ and 1 male (17\%). Their ages ranged from 36 to 70 years, with an average age of 53.2 years $(\mathrm{SD} \pm 13.9)$. The precranioplasty pathology was variable and included convexity meningioma (1 case), astrocytoma (2), ruptured arteriovenous malformation (1), suprasellar hemangiopericytoma (1), and traumatic intracerebral hemorrhage (1; Table 1). Application of the DermaClose RC device resulted in, on average, a $16 \%$ increase in the scalp surface area (range $6.57 \%-34.97 \%$ ) or a $13 \%$ increase in the scalp volume (range 3.64\%-29.57\%; Table 2). All reconstructions were completed within 7-10 days after placement of the external tissue expander. None of the patients required extensive ro- 


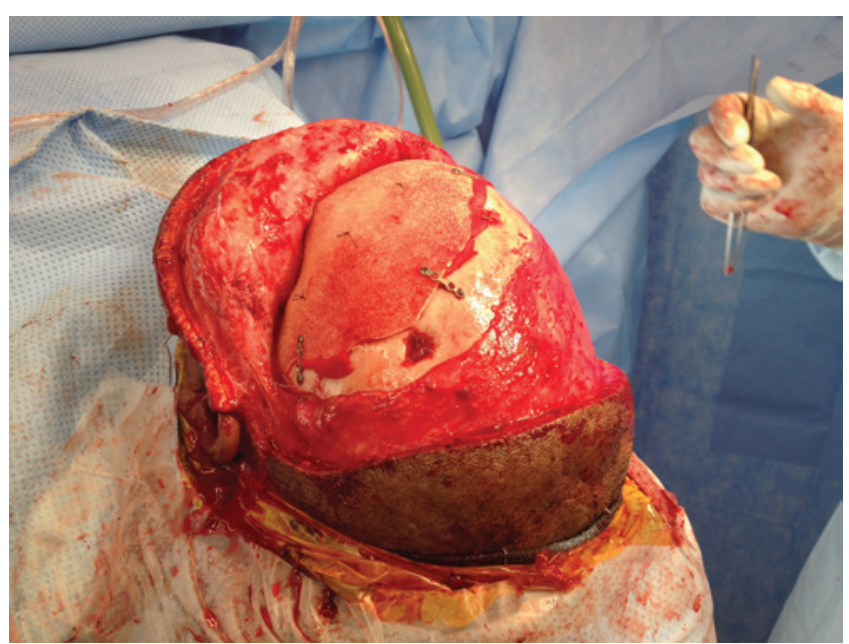

FIG. 3. Definitive cranioplasty with 3D-reconstructed synthetic graft. Note the tacking sutures placed to prevent epidural fluid collection. Figure is available in color online only.

tation flaps or skin grafts. All patients had adequate tissue for tension-free closure despite the increase in the cranial vault. The device was well tolerated, with all patients reporting pain scores $\leq 2$ according to the visual analog scale for pain. ${ }^{17}$ Short- and long-term follow-ups after cranial reconstruction revealed satisfactory wound closure and excellent cosmetic results in $100 \%$ of the patients. There were no cases of delayed wound closure, infection (including soft tissue, meningitis, or intracranial abscess formation), dermatitis, or cerebrospinal fluid leakage. All patients expressed satisfaction with their cosmetic outcome.

\section{Illustrative Cases \\ Case 1}

A 36-year-old female with history of 3 craniotomies for the resection of anaplastic astrocytoma, cosmetic methylmethacrylate cranioplasty, and external beam radiation therapy (EBRT) with concurrent chemotherapy underwent a fourth craniotomy for washout of subgaleal empyema and removal of a suspicious bone flap. After completing a course of antibiotics, the patient, while under local anesthesia, underwent delayed DermaClose RC device placement (180 days after bone flap removal) by a plastic surgeon in an operating room. Ten days after placement of the tissue expander, the patient underwent definitive cra- nioplasty without any complications. She was discharged to home 5 days after the reconstructive procedure. Followup 17 weeks after surgery revealed satisfactory wound closure and cosmetic results (Fig. 4).

\section{Case 2}

A 63-year-old diabetic female with history of decompressive craniectomy for a ruptured arteriovenous malformation followed by delayed cranioplasty underwent multiple craniotomies for wound dehiscence and a second craniectomy for removal of an infected bone flap. After completing a rehabilitation course, the patient, while under local anesthesia, underwent placement of a DermaClose RC expander in an operating room (235 days after bone flap removal). Ten days after placement of the tissue expander, she underwent definitive cranioplasty and was discharged to home 4 days later. Follow-up 7 weeks after surgery revealed excellent cosmetic results and no infectious complications (Fig. 5).

\section{Case 3}

A 67-year-old female with a history of 2 bifrontal craniotomies for the resection of suprasellar hemangiopericytoma followed by EBRT underwent a third craniectomy to remove the infected bone flap. After completing a course of antibiotics, the patient tolerated the placement of a DermaClose RC expander in the operating room while under local anesthesia (315 days after bone flap removal). Ten days after placement of the expander, she underwent definitive cranioplasty without complications. She was discharged to home 3 days after reconstructive surgery. Long-term follow-up 12 months after cranial reconstruction revealed excellent cosmetic results and no infectious stigmata (Fig. 6).

\section{Discussion}

There is archeological evidence that Incan surgeons were routinely performing trephinations of the cranium as early as $3000 \mathrm{BC}$ and that a minority of their patients also underwent cranioplasties. However, not until the 16th century did Fallopius describe the first case of cranial reconstruction with a gold plate. ${ }^{24}$ Almost a century later in 1668 in Amsterdam, van Meekeren successfully performed a cranioplasty using a dog's calvarial graft. ${ }^{24}$ Since then, a multitude of materials and techniques have been used for cranial reconstruction. ${ }^{27}$ Despite modern advances in aseptic and surgical techniques, cranial reconstruction remains

TABLE 1. Summary of patient demographics, primary pathologies, and complicating postoperative factors*

\begin{tabular}{ccclccccc}
\hline Case No. & Sex & Age $(\mathrm{yrs})$ & Primary Pathology & Postop XRT & No. of Surgeriest & $\Delta \mathrm{t}$ (days) & Days in Hospital & FU (days) \\
\hline 1 & F & 36 & Anaplastic glioma & Yes & 4 & 180 & 5 \\
\hline 2 & F & 63 & bAVM & No & 4 & 235 & 4 \\
\hline 3 & F & 62 & Hemangiopericytoma & Yes & 3 & 315 & 3 & 47 \\
\hline 4 & F & 49 & Anaplastic glioma & Yes & 6 & 278 & 1 & 5 \\
\hline 5 & M & 70 & Traumatic ICH & No & 3 & 262 & 6 \\
\hline 6 & F & 39 & Meningioma & No & 3 & 157 & 1 \\
\hline
\end{tabular}

$\mathrm{bAVM}=$ brain arteriovenous malformation; $\mathrm{FU}=$ follow-up; $\mathrm{ICH}=$ intracerebral hemorrhage; $\mathrm{XRT}$ = radiation therapy; $\Delta \mathrm{t}=$ time from bone flap removal to insertion of DermaClose RC device.

* All patients had postoperative infection.

$\dagger$ Number of craniectomies and/or craniotomies prior to application of DermaClose RC external tissue expander device. 
TABLE 2. Expansion of scalp surface area and volumes achieved with DermaClose RC device

\begin{tabular}{cccccccc}
\hline Case No. & DSSA $\left(\mathrm{mm}^{2}\right)$ & SSSA $\left(\mathrm{mm}^{2}\right)$ & $\%$ Increase in SSA & DSV $\left(\mathrm{mm}^{3}\right)$ & SSV $\left(\mathrm{mm}^{3}\right)$ & $\%$ Increase in SV & TVE Due to Implant $\left(\mathrm{mm}^{3}\right)$ \\
\hline 1 & 12,853 & 17,078 & 34.97 & 50,150 & 64,980 & 29.57 & 167,134 \\
\hline 2 & 9,664 & 10,377 & 7.38 & 46,951 & 49,359 & 5.13 & 64,576 \\
\hline 3 & 9,202 & 9,807 & 6.57 & 44,959 & 46,778 & 3.64 & 56,000 \\
\hline
\end{tabular}

SSA = scalp surface area; SV = scalp volume; TVE = total volume of tissue expansion.

a challenge for plastic surgeons and neurosurgeons alike. Reconstruction is especially difficult in patients with prior infections, cranial radiation, or a history of multiple craniotomies as the scalp will have been subject to significant scarring and contraction, making a procedure with a high morbidity $(16.4 \%)^{6}$ even more complicated.

It is our practice to delay all routine cranial reconstructions for 3-6 months after the initial neurological injury to allow for optimal recovery and minimize the risk of perioperative infection. The challenge in delaying cranial reconstruction following craniectomy is that scalp contraction is proportionately compounded over time, reducing the scalp surface area as well as volume and complicating the ability to create a durable and tension-free primary repair. The scalp of neuro-oncology patients who have received cranial radiation is especially difficult as ionizing radiation stimulates dense fibrosis leading to scalp atrophy and devascularization, a process that delays wound healing and promotes ulcer formation..$^{10,30}$ These challenges prompted the senior author (H.P.H.), who has had success with DermaClose RC in the closure of fasciotomies, to use this device to facilitate scalp tissue expansion in patients with large cranial defects and severe tissue retraction, which ultimately simplifies cranial reconstructions. While DermaClose RC use has been described for tissue expansion in the plastic surgery literature, ${ }^{2,11,20,25}$ there are no reports of its use for preoperative scalp tissue expansion prior to cranioplasties.
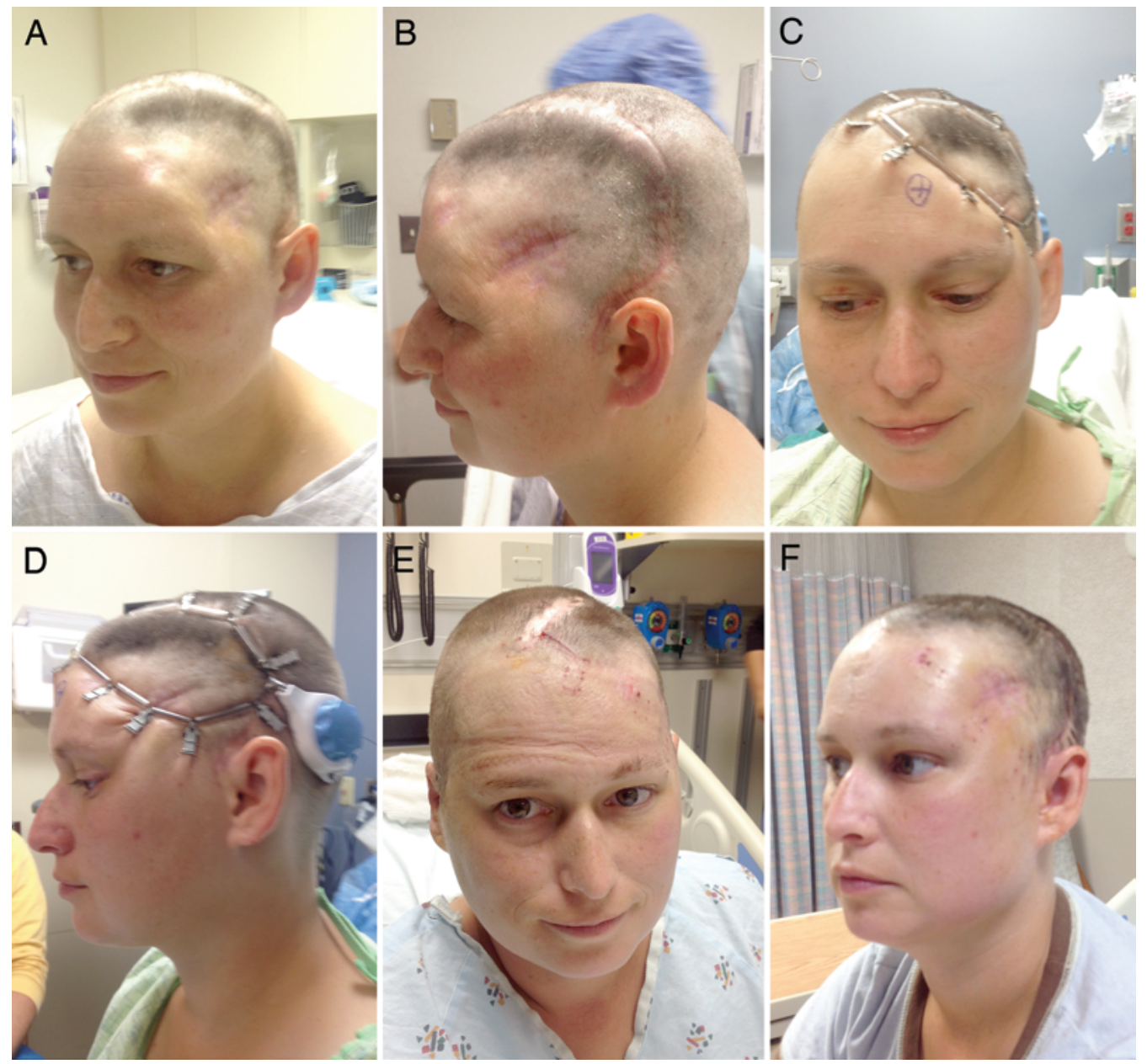

FIG. 4. Case 1. A 36-year-old female with recurrent glioma underwent multiple craniotomies and/or craniectomies, as well as EBRT, resulting in a large bony defect, scarring, and tissue retraction (A and B). The DermaClose RC device was applied to the scalp outside the margins of the bony defect (C and D). Postoperative images reveal good structural integrity and cosmetic results (E and F). Figure is available in color online only. 

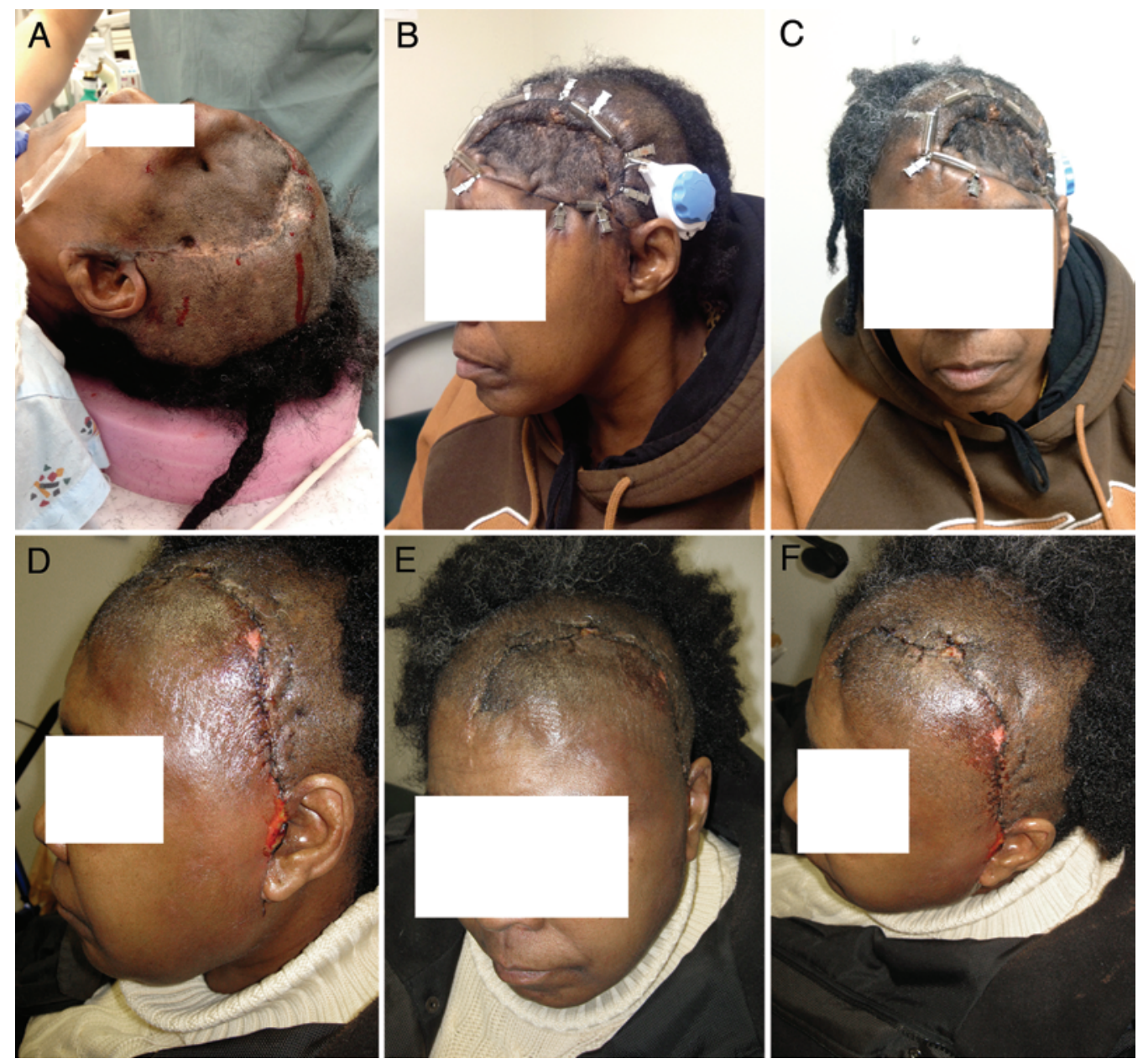

FIG. 5. Case 2. A 63-year-old female underwent multiple craniotomies and/or craniectomies for a ruptured arteriovenous malformation. Tissue scarring compounded by multiple scalp infections resulted in significant tissue retraction and cosmetic defect (A). Application of the DermaClose RC device facilitated scalp tissue expansion over the course of 10 days with minimal discomfort to the patient (B and $\mathbf{C})$. Postoperative images after definitive cranioplasty reveal good cosmetic results with no structural complications or infection (D-F). Figure is available in color online only.

The DermaClose RC device is placed circumferentially around the cranial defect, achieving tissue expansion without the need for traditional buried inflatable implants. Use of the DermaClose RC device is especially beneficial in patients with a history of soft tissue infections because, in contrast to the traditional internal tissue expanders, external scalp tissue stretch is not associated with potential contamination of the tissue planes used for the eventual cranioplasty. Other important advantages of this technique over buried implants include the following: 1) simplicity, because the device can be easily placed in an outpatient setting with the patient under local anesthesia; 2) costeffectiveness, because the device is less expensive than implanted devices; ${ }^{28} 3$ ) acceleration of wound closure because the device simply stretches the tissues back to their natural dimensions and eliminates the time required for cellular growth (7-10 days as opposed to 207 days of internal tissue expansion prior to definitive closure, reported in some studies);28 4) expeditious wound closure that allows patients to continue postoperative radiation therapy in a timely fashion; 5) eliminates the need for frequent outpatient visits for inflation, which are required for place- ment of traditional implantable devices; and 6) the device is well tolerated with minimal discomfort. ${ }^{25}$

Our reported technique has several noteworthy limitations. First, excessive soft tissue expansion over the curvature of the skull, in particular with a crisscrossing pattern of tension bands, is a significant limitation of the DermaClose RC device as the tension bands could lacerate the scalp even with the addition of protective plastic sleeves. This is the primary reason why we elected to apply the tension bands circumferentially around the defect. Second, while previous investigators have been able to calculate 2D measurements such as wound surface area before and after application of an external tissue expansion system, ${ }^{25}$ to date there are no validated reports of 3D software capable of accurately measuring the volume of expanded soft tissue after maximal scalp stretching in patients undergoing custom cranioplasty. This, coupled with the fact that our patients do not routinely undergo postcranioplasty imaging studies, forced our engineers to create a new method that calculates the volume of expanded scalp tissue based on measurements extrapolated from the unadulterated contralateral scalp as well as 3D soft-tissue renderings of each 

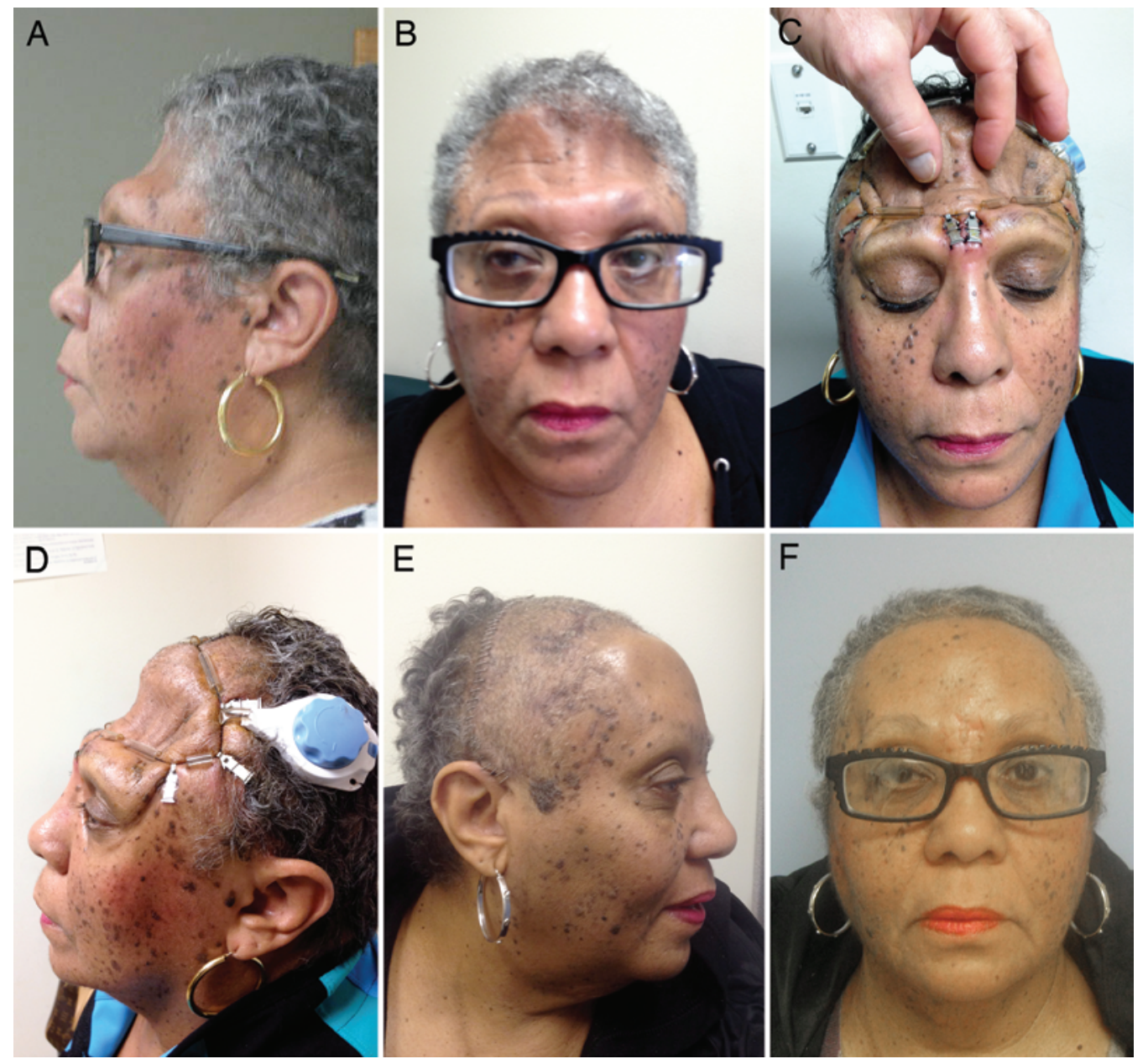

FIG. 6. Case 3. A 67-year-old female with recurrent hemangiopericytoma underwent multiple craniotomies and/or craniectomies and EBRT, resulting in reduced scalp volume and cosmetic defect (A and B). Application of the DermaClose RC device led to scalp tissue expansion in only 10 days ( $C$ and D). Postoperative images reveal good cosmetic results ( $E$ and F). Figure is available in color online only.

patient's head. Thus, there may be operator-dependent as well as software-dependent errors inherent in our calculations that may have under- or overestimated our final measurements. It is also important to note that a second company that supplied custom cranial implants for 3 of the patients in this study was unable to calculate scalp volumes despite employing sophisticated computer software. As a result, we could report changes in scalp volumes following the application of the DermaClose RC device in only 3 of the 6 patients. We hope that our novel approach can spark the creation of advanced software that can more easily measure scalp tissue volumes in the future.

The DermaClose RC device has broad applicability for the complex wound closures commonly seen in patients with devitalized tissues after infection, chemotherapy, and cranial radiation, as demonstrated by the patients in our series. The device's simplicity and durability obviates the need for resource-intensive and cumbersome free flaps or fragile skin grafts that are cosmetically unappealing and can be associated with greater overall risks of infection as well as higher levels of patient discomfort. ${ }^{20,23}$ Additionally, the DermaClose RC device is reliable, is associated with excellent cosmetic outcomes, and may prove to be more cost-effective than traditional implantable devices. Long-term prospective comparisons are necessary to determine the superiority of the DermaClose RC device over more traditional tissue expanders.

To date, no consensus guidelines exist for the management of complex cranial reconstructions, and large-scale randomized comparative studies are required to determine the efficacy of cranial reconstructions using this novel technique. However, our experience has been encouraging, and our technique has provided patients with excellent cosmetic results.

Regardless of the tissue expansion and/or mobilization technique used, the primary goal of any cranial reconstruction is to provide excellent structural and cosmetic results while minimizing morbidity. In select patients with complicating factors such as infected and discarded bone flaps, the volume generated by expensive prefabricated implants makes a primary, tension-free scalp closure even more imperative. While this series of 6 patients composes a small cohort, we believe that the reliability and technical ease of this method combined with an excellent structural success rate and the absence of morbidity in a complicated subset of patients makes the DermaClose RC device worth 
consideration in the reconstruction of large and complicated cranial defects.

\section{Conclusions}

Reconstruction of complex cranial defects remains a challenge for plastic surgeons and neurosurgeons despite advances in bioregenerative technology and surgical techniques. The DermaClose RC external scalp tissue expander allows for simple and effective cranial reconstruction in scenarios previously fraught with high complication rates.

\section{References}

1. Aydin S, Kucukyuruk B, Abuzayed B, Aydin S, Sanus GZ: Cranioplasty: Review of materials and techniques. J Neurosci Rural Pract 2:162-167, 2011

2. Bajoghli AA, Yoo JY, Faria DT: Utilization of a new tissue expander in the closure of a large Mohs surgical defect. J Drugs Dermatol 9:149-151, 2010

3. Bender A, Heulin S, Röhrer S, Mehrkens JH, Heidecke V, Straube A, et al: Early cranioplasty may improve outcome in neurological patients with decompressive craniectomy. Brain Inj 27:1073-1079, 2013

4. Blackwell KE, Rawnsley JD: Aesthetic considerations in scalp reconstruction. Facial Plast Surg 24:11-21, 2008

5. Bonfield CM, Kumar AR, Gerszten PC: The history of military cranioplasty. Neurosurg Focus 36(4):E18, 2014

6. Chang V, Hartzfeld P, Langlois M, Mahmood A, Seyfried D: Outcomes of cranial repair after craniectomy. J Neurosurg 112:1120-1124, 2010

7. Coulter IC, Pesic-Smith JD, Cato-Addison WB, Khan SA, Thompson D, Jenkins AJ, et al: Routine but risky: a multicentre analysis of the outcomes of cranioplasty in the Northeast of England. Acta Neurochir (Wien) 156:1361-1368, 2014

8. De Bonis P, Pompucci A, Mangiola A, D’Alessandris QG, Rigante L, Anile C: Decompressive craniectomy for the treatment of traumatic brain injury: does an age limit exist? J Neurosurg 112:1150-1153, 2010

9. De Filippo RE, Atala A: Stretch and growth: the molecular and physiologic influences of tissue expansion. Plast Reconstr Surg 109:2450-2462, 2002

10. Dormand EL, Banwell PE, Goodacre TEE: Radiotherapy and wound healing. Int Wound J 2:112-127, 2005

11. Durden F Jr, Tiwari P, Kocak E: Can the DermaClose device contribute to periwound tissue ischemia and necrosis: a case presentation and discussion? Plast Surg Nurs 32:132-133, 2012

12. Gordon CR, Fisher M, Liauw J, Lina I, Puvanesarajah V, Susarla S, et al: Multidisciplinary approach for improved outcomes in secondary cranial reconstruction: introducing the pericranial-onlay cranioplasty technique. Neurosurgery 10 (Suppl 2):179-190, 2014

13. Gürlek A, Alaybeyoğlu N, Demir CY, Aydoğan H, Bilen BT, Oztürk A: Aesthetic reconstruction of large scalp defects by sequential tissue expansion without interval. Aesthetic Plast Surg 28:245-250, 2004

14. Honeybul S: Neurological susceptibility to a skull defect. Surg Neurol Int 5:83, 2014

15. Kasper EM, Ridgway EB, Rabie A, Lee BT, Chen C, Lin SJ: Staged scalp soft tissue expansion before delayed allograft cranioplasty: a technical report. Neurosurgery 71 (1 Suppl Operative): $15-21,2012$

16. Leedy JE, Janis JE, Rohrich RJ: Reconstruction of acquired scalp defects: an algorithmic approach. Plast Reconstr Surg 116:54e-72e, 2005

17. McCormack HM, Horne DJ, Sheather S: Clinical applications of visual analogue scales: a critical review. Psychol Med 18:1007-1019, 1988
18. Miyazawa T, Azuma R, Nakamura S, Kiyosawa T, Shima K: Usefulness of scalp expansion for cranioplasty in a case with postinfection large calvarial defect: a case report. Surg Neurol 67:291-295, 2007

19. Neumann CG: The expansion of an area of skin by progressive distention of a subcutaneous balloon; use of the method for securing skin for subtotal reconstruction of the ear. Plast Reconstr Surg (1946) 19:124-130, 1957

20. O'Reilly AG, Schmitt WR, Roenigk RK, Moore EJ, Price DL: Closure of scalp and forehead defects using external tissue expander. Arch Facial Plast Surg 14:419-422, 2012

21. Ozkan O, Coskunfirat OK, Ozgentas HE, Derin A: Rationale for reconstruction of large scalp defects using the anterolateral thigh flap: structural and aesthetic outcomes. J Reconstr Microsurg 21:539-545, 2005

22. Piedra MP, Nemecek AN, Ragel BT: Timing of cranioplasty after decompressive craniectomy for trauma. Surg Neurol Int 5:25, 2014

23. Reddy S, Khalifian S, Flores JM, Bellamy J, Manson PN, Rodriguez ED, et al: Clinical outcomes in cranioplasty: risk factors and choice of reconstructive material. Plast Reconstr Surg 133:864-873, 2014

24. Sanan A, Haines SJ: Repairing holes in the head: a history of cranioplasty. Neurosurgery 40:588-603, 1997

25. Santiago GF, Bograd B, Basile PL, Howard RT, Fleming M, Valerio IL: Soft tissue injury management with a continuous external tissue expander. Ann Plast Surg 69:418-421, 2012

26. Schuss P, Vatter H, Marquardt G, Imöhl L, Ulrich CT, Seifert V, et al: Cranioplasty after decompressive craniectomy: the effect of timing on postoperative complications. J Neurotrauma 29:1090-1095, 2012

27. Shah AM, Jung H, Skirboll S: Materials used in cranioplasty: a history and analysis. Neurosurg Focus 36(4):E19, 2014

28. Singh NK, Reaven NL, Funk SE: Cost comparison of immediate one-stage and tissue-expander breast reconstructions after mastectomy in commercially insured patients. Manag Care 22:36-43, 2013

29. Song J, Liu M, Mo X, Du H, Huang H, Xu GZ: Beneficial impact of early cranioplasty in patients with decompressive craniectomy: evidence from transcranial Doppler ultrasonography. Acta Neurochir (Wien) 156:193-198, 2014

30. Tibbs MK: Wound healing following radiation therapy: a review. Radiother Oncol 42:99-106, 1997

31. Wachter D, Reineke K, Behm T, Rohde V: Cranioplasty after decompressive hemicraniectomy: underestimated surgery-associated complications? Clin Neurol Neurosurg 115:1293-1297, 2013

32. Yadla S, Campbell PG, Chitale R, Maltenfort MG, Jabbour P, Sharan AD: Effect of early surgery, material, and method of flap preservation on cranioplasty infections: a systematic review. Neurosurgery 68:1124-1130, 2011

\section{Disclosures}

Dr. Lee is a consultant for Medtronic.

\section{Author Contributions}

Conception and design: Reinard, Houin. Acquisition of data: Reinard. Analysis and interpretation of data: Reinard. Drafting the article: Reinard, Zakaria. Critically revising the article: Reinard, Lee, Houin. Reviewed submitted version of manuscript: Rock, Reinard, Lee. Study supervision: Rock, Houin. Calculation of scalp surface areas and volumes: Qatanani.

\section{Correspondence}

Jack P. Rock, Department of Neurosurgery, K-11, Henry Ford Hospital, 2799 W. Grand Blvd., Detroit, MI 48202. email: jrock1@hfhs.org. 